\title{
Obstetrics and COVID-19 Experience: Challenges and Strategies Adapted by a Tertiary Care Hospital in a Developing Country
}

\author{
Shazia Fakhar ${ }^{1}$, Irram Asghar ${ }^{1}$, Muhammad Ashraf ${ }^{2}$, Ejaz Khan ${ }^{3}$ and Sumeyya Azam ${ }^{4}$ \\ ${ }^{1}$ Department of Obstetrics \& Gynecology, Shifa International Hospital, Islamabad, Pakistan \\ ${ }^{2}$ Department of Anesthesia, Shifa International Hospital, Islamabad, Pakistan \\ ${ }^{3}$ Department of Pediatrics, Shifa International Hospital, Islamabad, Pakistan \\ ${ }^{4}$ Shifa Clinical Research Center, Shifa International Hospital, Islamabad, Pakistan
}

\begin{abstract}
Novel coronavirus disease 2019 (COVID-19) pandemic has produced profound effects on the global healthcare systems including every specialty. Pregnant women are physiologically immunocompromised and may be affected more by the pandemic than the general population. New and unique challenges were encountered by hospitals and healthcare providers (HCPs) related to medical, surgical and strategic management involved in obstetric care. Data of obstetric patients presenting in labour and delivery unit from April to July 2020 was studied. In all 533 patients presented, 46 (8.6\%) were COVID-19 positive. Overall, $41 \%$ cesarean sections were performed with one mortality and one COVID-19-positive neonate. The challenges faced by HCPs regarding delivery of care and collective successive measures taken to overcome those challenges as well as challenges faced by patients related to healthcare provision are also addressed. Strategies adopted for timely intervention to overcome those challenges were implemented for smooth and safe care to all patients and staff. We believe that planning and preparing proactively is the key to address any pandemic like COVID-19.
\end{abstract}

Key Words: COVID-19, Pregnancy, Obstetric care, Challenges.

How to cite this article: Fakhar S, Asghar I, Ashraf M, Khan E, Azam S. Obstetrics and COVID-19 Experience: Challenges and Strategies Adapted by a Tertiary Care Hospital in a Developing Country. J Coll Physicians Surg Pak 2021; 31(JCPSPCR):CR112-CR116.

\section{INTRODUCTION}

Novel coronavirus disease 2019 (COVID-19) pandemic has disrupted health and healthcare systems worldwide. Mostcountries have still not recovered from the immediate health effects imposed by this pandemic with increased mortality and morbidity due to severe acute respiratory syndrome coronavirus 2 (SARS-CoV-2).

According to UNICEF, five million babies are expected to be born in Pakistan in 9 months since COVID-19 pandemic declaration. ${ }^{1}$ With about 29 million expected births in South Asia during this pandemic. Pakistan holds the second place amongst the countries having the highest number of expected births. ${ }^{1}$ This means that new mothers and newborn babies will face the harsh realities of global containment measures including lockdowns and curfew, overwhelmed healthcare centres with supply and equipmentshortages and lack of sufficient skilled healthcare workers (HCWs).

Correspondence to: Dr. Shazia Fakhar, Department of Obstetrics \& Gynecology, Shifa International Hospital, Islamabad, Pakistan

E-mail:drshaziaf@yahoo.com

Received: December 13, 2020; Revised: January 29, 2021; Accepted: May 04, 2021

DOI: https://doi.org/10.29271/jcpsp.2021.JCPSPCR.CR112
Pregnant population is a vulnerable population, initially thought to be at increased risk of contracting this virus than the general population, but various studies since then have proven otherwise. ${ }^{2}$ It is reassuring to know that pregnant women with no underlying conditions do not appear more likely to contract the infection or become seriously ill than the general population. One study showed similar COVID-19 severity in pregnant patients $(86.0 \%$ mild disease, 9.3\% severe disease and $4.7 \%$ critical disease) to that reported in non-pregnant patients. ${ }^{3}$ However, increased severity of the disease has been observed in pregnancy in women with risk factors including increased body mass index (BMI) $>30$, age $>35$ years, BAME (Black, Asian, Ethnic Minority), with comorbidities like diabetes and hypertension ${ }^{4}$ or if infection occurs during the third trimester. ${ }^{5}$ Vertical transmission is still a controversial topic as initially most of the swabs taken from the placenta, amniotic fluid and nasopharyngeal swabs from the newborns were negative indicating no vertical transmission. ${ }^{6}$ However, there is emerging evidence that vertical transmission is possible with the evidence of presence of $\mathrm{IgM}$ antibodies in the newborn babies. ${ }^{7}$ After delivery, breastfeeding could be a route of transmission but evidence suggests otherwise, so it is reassuring that the COVID-19 positive mothers can breast-feed their babies while observing general precautions. They must be wearing a facemask, do frequent hand washing, follow respiratory hygiene and minimal handling of the baby to reduce chances of transmission of SARS-CoV-2 to theirbabies. 


\section{Site experience:}

As a tertiary care facility, we faced a huge responsibility to provide best obstetric care, keeping in view the safety and precautions for both the patients and the HCWs.

Data from Obstetric Department from April to July 2020, showed considerable rise in the number of COVID-19 positive pregnant patients, most prominent in the month of June post-Eid-ul-Fitr festival (Figure 1). Most of the patients were asymptomatic. The symptomatic patients had mild symptoms and their management and mode of delivery was according to the obstetrics indications (Table I). All patients were conservatively managed and most had uneventful recovery. However, there was a single mortality of a 20-week pregnant lady, who had severe thrombotic thrombocytopenic purpura (TTP). She was managed with multiple platelet transfusions and plasmapheresis and finally underwent splenectomy. Unfortunately, she ended up having a fetal demise followed by maternal death. The cause of her death was TTP, complicated by superimposed COVID-19. Thus, we witnessed a single mortality in the past four months at our facility compared with COVID-19 associated maternal mortality rate of 5.6 per 100,000 maternities in UK. ${ }^{8}$ There was a single COVID-19 positive baby born via normal vaginal delivery to a COVID-19 positive mother.

\section{Challenges faced and strategies adapted:}

This pandemic imposed a lot of challenges to existing institutional practices which warranted timely interventions and develop new strategies to overcome them. A number of steps were taken including closure of outpatient departments (OPDs) and some inpatient areas, discontinuing all elective surgeries and procedures, limiting entry and exit areas within the hospital, designating triage areas, starting surveillance (pre-surgical COVID-19 screening), and developing guidelines for COVID-19 diagnosis, admission, exposure and management using national and international updated evidence.

\section{Challenges related to the doctors and strategies:}

With the rapid spread of COVID-19 across the world including Pakistan, there was a sudden increase in the demand and supply of personal protective equipments (PPEs). ${ }^{9}$ Initially, the PPEs were used wisely in high alert areas including the designated Red Zones (emergency room and critical care units). Labour and delivery unit was also deemed as the vulnerable area where patients come in emergency and many a times have to be rushed for delivery or a C-section without knowing their COVID status, provision of PPEs including N-95 facemasks, hazmat suits and face shields, was ensured for all HCPs in every shift.

Maintaining social distancing was difficult among the HCPs working within the labour and delivery area, including the resting and dining areas within the labor and delivery suites. Standard operating procedures (SOPs) were rapidly developed for these areas. All HCPs were advised not to share meals, avoid clustering in the confined places, avoid travel together and new designated rest rooms were identified to ensure social distancing.

Resident training programme was also compromised during this period as all academic classes, mortality and morbidity meetings as well as tumor boards, were suspended. OPDs were closed, elective gynecological surgeries were postponed and workload plummeted to low levels. However, we resumed our academic meetings online via zoom application for the continued training and education of our residents and staff.

Roster adjustment became a cumbersome job due to exposure of our HCPs to COVID-19 suspected or positive patient or a family member. This challenge was overcome by dividing the whole team of doctors into three groups, each working in $1 / 3$ roster for a week followed by rest period of 2 weeks to ensure quarantine time for the symptoms to appear.

Mental health became an emerging issue during this pandemic. For all the HCPs, including doctors and nursing staff, it was one of the biggest challenges as most developed severe anxiety and fear of getting infected by the virus and taking infection back home to their families and loved ones. Education regarding emerging new situations, teaching them how to protect themselves, counseling them timely, building their confidence in those difficult times was a great challenge faced by all the doctors especially in the high alert zones. The news from the social media regarding the rising numbers, continued exposure and deaths of the fellow doctors across Pakistan added to this stress. Uncertainty at national and international levels, regarding many aspects of the disease, further aggravated the situation. Family and the peer pressure on HCPs led to many of them quitting their jobs. To deal with these psychological strains, virtual seminars and sessions were arranged in collaboration with Employee Health Clinic. Live sessions were conducted by the qualified team of psychologists and psychiatrists who were actively involved in promoting these sessions and conducting face-to-face consultations.

As antenatal care is an ongoing process, it was very important to give standard care to pregnant patients while minimising the exposure to the HCPs. To facilitate this, we adopted telemedicine clinics and hybrid clinics for those patients who needed any medical advice or needed to be seen by the consultant in personaccordingly. ${ }^{8}$

Making a strategy for the HCPs post-exposure to a COVID-19 suspected or positive patient was a great challenge for which standard protocols were laid down. Post-exposure measures included contact tracing, immediate disinfection of the area by involving the infection control team, immediate reporting to the employee health clinic, quarantine of the exposed HCWs according to their risk assessment, adjustment of the rosters, taking care of their mental health, monitoring, follow-up and formulation of back-to-work policy. 
Table I: Frequency and percentage for outcome of COVID-19 positive cases from April to July 2020 in Obstetrics \& Gynecology Department of the Hospital.

\begin{tabular}{|c|c|c|c|c|c|c|c|c|c|c|c|}
\hline & $\begin{array}{c}\text { Total } \\
\text { COVID } \\
\text { samples }\end{array}$ & $\begin{array}{l}\text { Total } \\
\text { COVID } \\
\text { positive } \\
\text { cases }\end{array}$ & $\begin{array}{l}\text { Symptom--atic } \\
\text { cases }\end{array}$ & $\begin{array}{l}\text { Asymptom- } \\
\text {-atic cases }\end{array}$ & $\begin{array}{l}\text { Conservative } \\
\text { management }\end{array}$ & $\begin{array}{l}\text { Spontaneous } \\
\text { vaginal } \\
\text { delivery } \\
\text { (SVD) }\end{array}$ & $\begin{array}{l}\text { Elective } \\
\text { lower } \\
\text { uterine } \\
\text { segment } \\
\text { C-section } \\
\text { (LSCS) }\end{array}$ & $\begin{array}{l}\text { Emergency } \\
\text { LSCS }\end{array}$ & $\begin{array}{c}\text { ICU } \\
\text { admissions }\end{array}$ & Discharges & $\begin{array}{c}\text { COVID } \\
\text { positive } \\
\text { babies }\end{array}$ \\
\hline $\begin{array}{l}\text { April } \\
2020\end{array}$ & 73 & 0 & NA & NA & NA & NA & NA & NA & NA & NA & NA \\
\hline $\begin{array}{l}\text { May } \\
2020\end{array}$ & 113 & $\begin{array}{c}10 \\
(8.8 \%)\end{array}$ & $\begin{array}{c}3 \\
(2.6 \%)\end{array}$ & $\begin{array}{c}7 \\
(6.2 \%)\end{array}$ & 0 & $\begin{array}{c}7 \\
(6.2 \%)\end{array}$ & 0 & $\begin{array}{c}3 \\
(2.6 \%)\end{array}$ & 0 & $\begin{array}{c}113 \\
(100 \%)\end{array}$ & $\begin{array}{c}1 \\
(0.8 \%)\end{array}$ \\
\hline $\begin{array}{l}\text { June } \\
2020\end{array}$ & 190 & $\begin{array}{c}19 \\
(10 \%)\end{array}$ & $\begin{array}{c}5 \\
(2.6 \%)\end{array}$ & $\begin{array}{c}14 \\
(7.3 \%)\end{array}$ & $\begin{array}{c}6 \\
(3.1 \%)\end{array}$ & $\begin{array}{c}5 \\
(2.6 \%)\end{array}$ & $\begin{array}{c}6 \\
(3.1 \%)\end{array}$ & $\begin{array}{c}2 \\
(1.1 \%)\end{array}$ & $\begin{array}{c}1 \\
(1.1 \%)\end{array}$ & $\begin{array}{c}189 \\
(99.47 \%)\end{array}$ & 0 \\
\hline $\begin{array}{l}\text { July } \\
2020\end{array}$ & 157 & $\begin{array}{c}17 \\
(10.2 \%)\end{array}$ & 0 & $\begin{array}{c}17 \\
(10.2 \%)\end{array}$ & $\begin{array}{c}1 \\
(0.6 \%)\end{array}$ & $\begin{array}{c}8 \\
(3.7 \%)\end{array}$ & $\begin{array}{c}3 \\
(1.9 \%)\end{array}$ & $\begin{array}{c}5 \\
(0.63 \%)\end{array}$ & 0 & $\begin{array}{c}157 \\
(100 \%)\end{array}$ & 0 \\
\hline
\end{tabular}

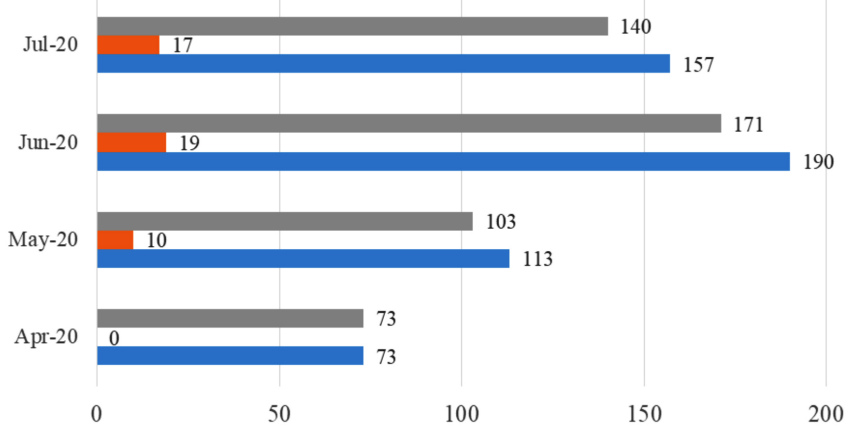

๓COVID-19 Negative cases $n$ COVID-19 Positive cases $\approx$ Total COVID-19 samples

Figure 1: Frequency of COVID-19 positive and negative cases from April to July 2020, in Obstetrics \& Gynecology Department of the Hospital.

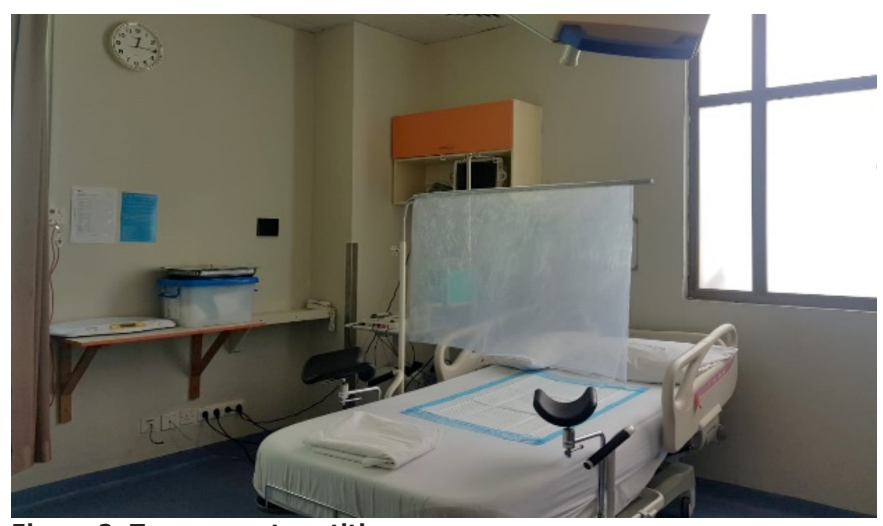

Figure 2: Transparent partition screen.

Challenges and strategies for doctor-patient interactions:

There were certain challenges faced by the doctors in relation to patients. Most common was non-adherence to the SOPs as many patients and their attendants were not compliant. Provision of masks was made mandatory at the entrance of the hospital for all the patients and their attendants entering the hospital premises.

Initially, most patients failed to divulge their symptoms, contact and travel history or COVID-19 status. This was partly because of the fear of being put in government quarantine centres or the social stigmatisation that became so common. Detailed and targeted history questionnaire was evolved to avoid missing cases in the triage areas.

In OPD, we developed telemedicine clinics where tele-consultations were done via a video link at the convenience of patient's home, thus minimizing exposure of the patient and HCWs.

Hybrid clinics were established for those patients who needed to be seen physically by the consultants. In order to minimise exposure, patient's history was taken on video or phone links followed by examination in a separate room with full PPEs. However, it created a new problem when large number of patients started seeking hybrid appointments and coming in the clinics in clusters where maintaining social distancing in the waiting areas was compromised. This was addressed by a strict check on unnecessary appointments and hybrid appointments were given only on the request of the consultants to those patients who really needed in-person consultation. Rest of the consultations was done on telemedicine.

All the pre-surgery assessments were done on the outpatient basis via telemedicine, especially anesthesia assessment. COVID-19 PCR testing was made mandatory for all urgent surgeries and induction of labor planned at term. This strategy proved to be very useful as it gave confidence and support to the HCPs as well.

Lengthy turnaround time (TAT) for COVID-19 PCR test reports was another challenge. This was solved by increasing the number of slots and batches per day thus decreasing TATs significantly to five hours only. This strategy not only remarkably expedited medical care and disposition of the patients according to their COVID-19 status but also minimized exposure of our HCPs.

Meticulous work was done by the Obstetric Department in collaboration with administration in evolving protocols for patient admission and disposition. In case of emergency, where COVID-19 status was undetermined, nasopharyngeal swab for COVID-19 PCR was taken, patient was kept in isolation room and delivery was done in designated negative pressure delivery suites or operated upon in designated negative pressure operating rooms, according to their 
obstetric needs. ${ }^{10}$ Post-procedure such patients were shifted to the designated isolated rooms or to what was called the clinical decision unit till their results were confirmed. In case of positive result, the patient was shifted to designated negative pressure COVID-19 Unit, evaluated and treated in consultation with other disciplines including infectious diseases and critical care, if needed. Those who had negative results were shifted to the regular floors for routine care. Most of these patients were treated by our multidisciplinary teams.

Proper sampling of COVID-19 PCR nasopharyngeal swab training was given to all doctors and paramedical staff. Similarly, donning and doffing drills were conducted for HCPs. We divided our labour room into COVID-19 negative area and COVID-19 suspects area. Separate delivery suites were designated for the COVID-negative patients who were delivered in positive pressure rooms. Patients highly suspected of having a COVID-19 infection or COVID-19-positive patients were delivered in negative pressure delivery suites. In order to minimize the staff exposure to COVID-19-positive patients, designated doctor and nurse were assigned in the delivery room for labor monitoring and delivery and early discharge was adopted as a general policy. A separate room was used for the neonatal team to receive the neonates of COVID-19-positive mothers in order to minimize the exposure of the neonatal team.

As the risk of aerosol infection was highest during parturition, a transparent screen was designed by our bio-medical department which had a water impermeable plastic curtain (Figure 2). Its mounting stands have adjustable height to ensure a physical barrier between the HCPs and the patient during delivery to minimize exposure.

Transfer of COVID-19-positive patients within the hospital for diagnostics like ultrasonography (USG), computed tomography $(\mathrm{CT})$ or magnetic resonance imaging (MRI) or to operating rooms was a tedious job. An organised pathway was designed in which the patient was transferred through designated corridors with the help of a designated team which included a HCP, a security guard in front to clear the path to avoid unnecessary contacts and a sanitary worker behind disinfecting the area, cleaning the elevator knobs and door handles promptly to minimize the spread of infection within the hospital.

Breaking bad news to the patient of being COVID-19 positive, triggered denial from the patients as most of them were asymptomatic and they wanted to leave hospital against medical advice immediately. Such patients were dealt with immediate and meticulous counseling by the residents and consultants.

To maintain the social distancing and decreasing the number of visitors to hospital, policy of "single and same attendant" with the patients was strictly enforced.
Being a tertiary care hospital, we used to accept referrals and non-booked patients from our twin cities and beyond including distant referrals. However, with increased influx of COVID-19 positive patients, a hospital policy was made to discontinue this practice so as not to overwhelm our existing bed capacity and to cater to only our booked patients unless there was a dire emergency to the mother or the fetus.

Plan of continuity of care for COVID-19 positive antenatal patients, requiring Elective Cesarean Sections, Elective/Urgent gynecological surgeries and return of COVID positive patients seeking medical care after being in quarantine was another challenge. Currently, elective gynecological surgeries were deferred for the COVID-19 positive patients till their PCR was negative.

\section{Challenges faced by the patients and strategies:}

The biggest challenge faced by the patients was an unnecessary increase in cesarean section rate due to this pandemic. Although studies have shown that the number of critical pregnant patients has been very low. One study found $4.7 \%$ of critical patients among all pregnant women but there has been a substantial increase in cesarean section rate to $59 \%{ }^{4}$ to $92 \% .^{11}$

Here at this facility, we are ensuring quality care, respecting the birth choices of the pregnant women as much as possible and decisions for cesarean sections are made solely on clinical grounds and obstetric indications. We observed an overall cesarean section rate of $41 \%$ during this pandemic with $19.5 \%$ elective and $21.7 \%$ emergency cesarean sections performed.

Understandably, most pregnant women were very anxious at this very unusual time due to concerns regarding their health and well-being of their babies, access and availability of their HCPs. To address this issue, administration organised a series of webinars accessible to not only HCPS but also to general public. Qualified obstetricians, gynecologists, anesthesiologists, pediatricians, and infectious diseases specialists shared their experiences and strategies to deal with pregnant population and their queries and concerns were addressed in live sessions. The main focus was to allay their fears about the disease, and to inform them about safety measures that were in place where safe obstetric care could be provided and access to continuity of care through telemedicine. Our media department made awareness videos and pamphlets about the disease and the precautions being in place within the hospital setting to minimize infection.

World Health Organization (WHO) has praised Pakistan's handling of the COVID-19 pandemic with effective strategies devised by the government of Pakistan as among one of the seven countries that world needs to learn from to deal with the pandemics like COVID-19. ${ }^{12}$ 
We strongly believe that planning and preparing proactively is the key to address any pandemic like COVID-19. Addressing specific issues in obstetric care about safety, diagnosis, management and follow-up care through guidelines, SOPs and easy accessibility, are keys to gain trust of our patients for optimal care and outcome.

\section{CONFLICT OF INTEREST:}

The authors declared no conflict of interest.

\section{AUTHORS' CONTRIBUTION:}

SF: Conception of idea, planning and organising the main features of the manuscript.

IA: Writing the manuscript.

MA: Data collection and review.

EK: Critical review of the manuscript.

SA: Formatting and reviewing the manuscript.

\section{REFERENCES}

1. UNICEF, Pakistan. Available at: www.un.org.pk. Retrieved September 13, 2020.

2. Guan W, Ni Z, Hu Y, Liang W, Ou C, He J, et al. Clinical characteristics of coronavirus disease 2019 in China. N Engl J Med 2020; 382:1708-20. DOI: 10.1056/NEJMoa2002032.

3. Breslin N, Baptiste C, Gyamfi-Bannerman C, Miller R, Martinez R, Bernstein K, et al. COVID-19 infection among asymptomatic and symptomatic pregnant women: Two weeks of confirmed presentations to an affiliated pair of New York City hospitals. Am J Obstet Gynecol MFM 2020; 2(2):100118. doi: 10.1016/j.ajogmf.2020. 100118.

4. Schwartz D, Dhaliwal A. Infections in pregnancy with COVID-19 and other respiratory RNA virus diseases are rarely, if ever, transmitted to the fetus: Experiences with coronaviruses, HPIL, hMPV RSV, and influenza. Arch Pathol Lab Med 2020; 144: 920-28. doi: 10.5858/arpa.2020- 0211SA.
5. Knight M, Vousden N, Simpson N, O'Brien P, Brocklehurst P. Characteristics and outcomes of pregnant women admitted to hospital with confirmed SARS-CoV-2 infection in UK: National population based cohort study. BMJ 2020; 369: m2107. doi: 10.1136/bmj.m2107.

6. Yu N, Li W, Kang Q, Xiong Z, Wang S, Lin X, et al. Clinical features and obstetric and neonatal outcomes of pregnant patients with COVID-19 in Wuhan, China: A retrospective, single-centre, descriptive study. Lancet Infect Dis 2020: 20(5); 559-64. doi: 10.1016/S1473-3099(20)30176-6.

7. Wang $\mathrm{C}$, Zhou $\mathrm{YH}$, Yang HX, Poon LC. Intrauterine vertical transmission of SARS-CoV-2: what we know so far. Ultrasound Obstet Gynecol 2020; 55(6): 724-725. doi: 10.1002/ uog. 22045.

8. Royal College of Obstetricians \& Gynaecologists. Coronavirus (COVID-19) Infection in Pregnancy. 2020. www.rcog.org.uk/en/guidelines-research-services/guidelines/coronavirus-pregnancy/. Retrieved September 13, 2020.

9. World Health Organization. Rational Use of Personal Protective Equipment for Coronavirus Disease (COVID-19) and Considerations during Severe Shortages. 2020. Available at: http://apps.who.int/iris/bitstream/handle/10665/ 331695/WHO-2019-nCov-IPC_PPE_use-2020.3-eng.pdf. Retrieved September 13, 2020.

10. Poon LC, Yang H, Dumont S, Lee JCS, Copel JA, Danneels L, et al. ISUOG Interim guidance on coronavirus disease 2019 (COVID-19) during pregnancy and puerperium: information for healthcare professionals-an update. Ultrasound Obstet Gynecol 2020; 55(6):848-62. doi: 10.1002/uog.22061.

11. Zaigham $M$, Anderrson $O$. Maternal and perinatal outcomes with COVID-19: A systematic review of 108 pregnancies. Acta Obstet Gynecol Scand 2020; 99(7):823-29. doi: 10.1111/ aogs.13867.

12. World Health Organization (WHO). Available at: http://www.who.int/dg/speeches/detail/who-director-general -s-opening-remarks-at-the-media-briefing-on-covid-19-7september-2020. Retrieved September 13, 2020. 